\title{
Umgangsformen mit der Unmöglichkeit
}

Der erste Teil dieser Reihe erläuterte die Paradoxie eines Spitalmanagements [1]. Hier geht es nun darum, in dieser Situation konkrete Möglichkeiten des Managements aufzuzeigen. Ein Beispiel ist das sogenannte «bilateral-situative» Entscheiden.

Harald Tuckermann ${ }^{a}$, Johannes Rüegg-Stürm ${ }^{b}$, Matthias Mitterlechner

a Prof. Dr. oec. HSG, Assistenzprofessor für Management pluralistischer Organisationen und Co-Leiter Forschungsprogramm HealthCare Excellence, Vize-Direkto des Instituts für Systemisches Management und Public Governance an der Universität St. Gallen

b Prof. Dr. oec. HSG, Direktor des Instituts fü Systemisches Management und Public Governance an der Universität St. Gallen

c Dr. oec. HSG, Habilitand und Co-Leiter Forschungsprogramm HealthCare Excellence an der Universität St. Gallen
Korrespondenz:

Prof. Dr. oec.

Johannes Rüegg-Stürm Institut für Systemisches Management und Public Governance Dufourstrasse 40a CH-9008 St. Gallen Tel. 0712242323 Fax 0712242536

johannes.rueegg[at]unisg.ch www.imp.unisg.ch/de/ Weiterbildung
Der erste Beitrag [1] hat dazu eingeladen, die Komplexität von Spitalmanagement als Paradoxie aufzufassen. Die Paradoxie entsteht, weil auf der einen Seite die Notwendigkeit zu organisationsweiten Entscheidungen zunimmt - Fragestellungen, die über die Klinik-, Bereichs-, Professions- und Abteilungsgrenzen hinausgehen. Gleichzeitig sind Spitäler historisch durch unterschiedliche Professionen und zunehmend spezialisierte ärztliche Disziplinen geprägt, die jeweils eigene und für ihre Behandlungspraxis adäquate Formen des Entscheidens entwickelt haben. Aus diesen beiden Polen der vielfältigen Entscheidungspraxis autonomer Teilbereiche und der zunehmenden organisationsweiten Entscheidungen entsteht die Paradoxie von Spitalmanagement. Management befasst sich zu einem wesentlichen Teil mit der Integration beider Pole. Die Abbildung 1 zeigt die Paradoxie von Management:

Dieser und der folgende Beitrag thematisieren zwei Stossrichtungen, um mit der Paradoxie von Spitalmanagement umzugehen $[2,3,4]$ : sogenannte «sowohl-als-auch»- und «weder-noch»-Ansätze. Bei beiden geht es nicht darum, sich entweder auf die eine Seite oder auf die andere Seite der Paradoxie zu schlagen, also entweder die Vielfalt zu unterdrücken oder die organisationsweiten Entscheidungen auszublenden. Vielmehr geht es bei diesen Ansätzen der Paradoxiehandhabung darum, auf je unterschiedliche Weise beide Seiten aufeinander zu beziehen. Letztlich sind spitalweites und disziplinenspezifisches Entscheiden beide für ein Spital notwendig.

\section{«Sowohl-als auch»: bilateral-situatives Entscheiden}

Die erste Stossrichtung besteht darin, sowohl die vielfältige Entscheidungspraxis relativ autonomer Bereiche als auch die organisationsweiten Entscheidungen im Blick zu haben. Diese Möglichkeit fokussiert vor allem auf das Verhältnis zwischen den beiden Seiten der Paradoxie. Sie ist durch bilaterale Absprachen der relativ autonomen Akteure charakterisiert, die situativ ihre unterschiedlichen Interessen koordinieren. Wir nennen diese Stossrichtung «bilateralsituatives Entscheiden».

\section{Gérer l'impossibilité}

Si l'on considère la gestion d'un hôpital comme la manifestation du paradoxe lié aux multiples formes de prises de décisions, la question se pose de savoir comment un hôpital peut malgré tout prendre des décisions communes. Une première possibilité est celle dite des décisions «bilatérales en fonction de la situation", qui met en avant des accords bilatéraux entre les différentes parties prenantes pour répondre à la nécessité décisionnelle qui apparaît en fonction de la situation. L'accent n'est donc plus mis sur les différences du paradoxe mais sur leur interaction.

Ein zentrales Moment ist hierbei das, was ein Geschäftsleitungsmitglied als Gärtchendenken beschreibt: «Jeder schaut nur auf sein Gärtchen: die Internisten, die Chirurgen. Die Verwaltung denkt primär ans Geld, was ja auch korrekt ist, und so weiter. Es ist eine Geschäftsleitung, die letztendlich doch mehr die Partikularinteressen vertritt.» Das bedeutet jedoch nicht, dass es nicht zu wechselseitiger Koordination und Abstimmung kommt, denn, so der Gesprächspartner weiter: «Es ist auch so: Man schaut wirklich, also man würde sich auch den Rasenmäher ausleihen. Man würde einmal die Rosen schneiden oder giessen. Gärtchen heisst ja: Du musst immer jemanden haben, der dafür sorgt, wenn du mal nicht da bist.» Das Gärtchendenken hebt die vielfältige Entscheidungspraxis, aber auch den wechselseitigen Bezug und die Abstimmungsmöglichkeiten hervor, also beide Seiten der Paradoxie.

Mit Blick auf die Gesamtorganisation folgt aus dem Gärtchendenken erstens, dass Entscheidungen in der Organisation verteilt sind. Es wird tendenziell unklarer, an welcher Stelle und von wem welche Ent- 
Abbildung 1

Die Paradoxie von Management von Spitälern.

\section{Paradoxie «multirationales Management»}

Ebene der Kliniken/Fachbereiche/Abteilungen vs. Ebene Gesamtspital

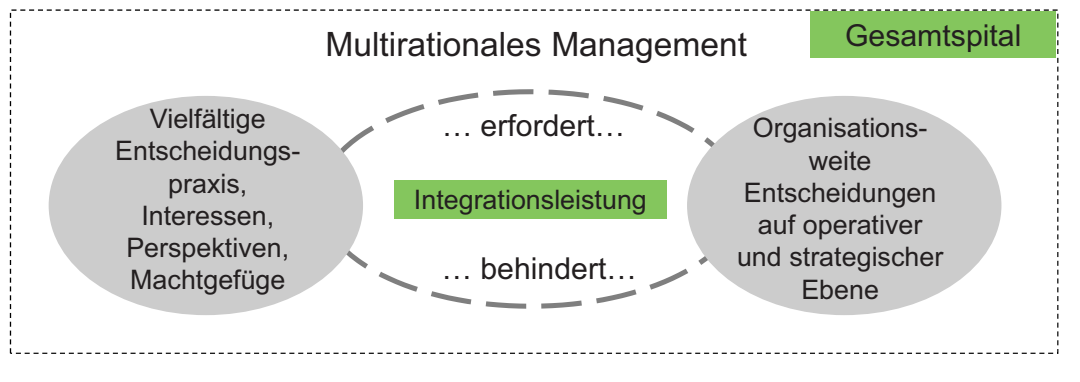

scheidungen getroffen werden. Das gilt vor allem für diejenigen Entscheidungen, die über die unterschiedlichen Gartenzäune hinweg gelten und wirken. Umgekehrt erfordert dies von denjenigen Stellen, die organisationsweite Entscheidungen verantworten, einen guten Ein- und Überblick über die Organisation. Entsprechend beschreibt ein Geschäftsleitungsvorsitzender die Rolle der Geschäftsleitung auch als «Sounding Board», in dem man selbst Themen einbringt und gleichzeitig auch herausfiltert, welche Themen in der Organisation aktuell sind.

\section{Autonome Akteure koordinieren ihre Interessen situativ in bilateralen Absprachen.}

Zweitens folgt aus dem Gärtchendenken, dass Entscheidungen auf Gesamtspitalebene vergleichsweise schwer gegen potentiell betroffene Bereiche gefällt werden können, sondern die Spitalleitung auf deren Zustimmung angewiesen ist [5]. Gleichermassen beobachtet ein Geschäftsleitungsmitglied eines Kantonsspitals ein grosses Harmoniestreben: «Das Harmoniebedürfnis ist hoch. Man versucht, Konsens $\mathrm{zu}$ erreichen. Man versucht, sich möglichst nicht in das andere Gebiet einzumischen.» Dieses sogenannte «Harmoniestreben» trägt zur Stabilisierung der Gesamtorganisation bei, ermöglicht es den Beteiligten, Konflikte und Differenzen räumlich und zeitlich zu verschieben. Konfliktbeladene Situationen werden weniger in der Öffentlichkeit eines Gremiums (wie der Geschäftsleitung), sondern eher in bilateralen Gesprächen zwischen einzelnen Klini- ken, Fachbereichen und Instituten gehandhabt. Einzelgespräche sind vergleichsweise leichter zu handhaben und erlauben dadurch überhaupt eine Einigung oder Entscheidung zwischen zwei Parteien. Demgegenüber sind grössere Gruppen wie die Geschäftsleitung durch die vielfältigen Interessen, wechselseitigen Verpflichtungen und Abhängigkeiten kaum zu steuern. Man kann mit Bilateralismus für Entscheidungen zu Einzelthemen sorgen, manchmal auch in hoher Geschwindigkeit. Zudem wird darüber die Autonomie der Fachbereiche nicht in Zweifel gezogen.

Drittens wird im bilateral-situativen Entscheiden die Handhabung organisationsweiter Fragen zu einer fortlaufenden Bewegung, die nur in begrenzter Form im Vorhinein planbar ist. Alles scheint im Prozess zu sein, wie ein Spitalleiter erläutert: «Die Kliniken sind da eher Selbstläufer (...) Bei unserer Vernetzung und wenn die Kliniken weiter unabhängig sind (...) Das läuft sowieso alles sehr viel über die informellen Netzwerke, da kennt man sich untereinander (...) Da ist grosse Flexibilität gefordert.»

Viertens erfolgt das Agenda-Setting - die Art und Weise, wie Themen in der Organisation zustande kommen - bei bilateral-situativen Entscheidungen eher bottom-up als top-down. Hierdurch kann eine Vielzahl von Entscheidungsnotwendigkeiten angeregt werden. Dabei ist die Klärung anspruchsvoll, welche Themen und Fragestellungen für das Spital insgesamt zentral und als Entscheidung zu bearbeiten sind. Das «Agenda-setting» erfolgt dezentral: «Die Anstösse müssen von den Einheiten kommen, man kann nicht von oben die Struktur bestimmen» (Geschäftsleitungsmitglied). Eine Folge davon ist, dass tendenziell eher bereichsspezifische Themen und weniger organisationsweite Entscheidungen zur Bearbeitung kommen. Eine weitere Folge kann darin bestehen, dass die anspruchsvolle und mitunter unwahrscheinliche Klärung der zahlreichen anstehenden Themen und Fragestellungen ihrerseits Ungewissheit und Ambiguität erzeugt, die selbst wiederum über bilaterale Absprachen abgearbeitet werden. Daraus zeichnet sich eine Vervielfältigung und zunehmende Verteiltheit von Entscheidungen ab. Letztlich können sich dann Entscheidungen verzögern, zumal gerade Entscheidungen auf Ebene der Gesamtorganisation eine Vielzahl unterschiedlicher bilateraler Vorbereitungsklärungen erfordern. «Ich muss in einer Vorphase mit jedem unter vier Augen sprechen. Ich darf bloss nicht mit allen zusammen sprechen, da gäbe es ein vereinigtes 〈Nein〉 ... Nach dieser Vorarbeit haben wir dann zusammen eine Sitzung» (Geschäftsleitungsmitglied).

Fünftens kann das bilateral-situative Entscheiden dazu tendieren, eher diejenigen Alternativen zu verfolgen, die weniger Widerspruch erwarten lassen. Eher konfliktbeladene Entscheidungen laden zu einer Verlagerung ein und geraten somit ins Abseits oder 
lösen verzögert Konflikte aus. So wird beispielsweise ein Beschluss zu Investitionen in die Zukunft verschoben oder die Abgrenzung eines ausgegliederten neuen interdisziplinären Zentrums an die operative Ebene verlagert. Die einen warten dann auf die $\mathrm{Zu}-$ sage ihrer Mittel, während die anderen sich mit Kompetenzstreitigkeiten aufhalten, die auf ihrer Ebene der täglichen Patientenbehandlung nicht grundsätzlich zu handhaben sind. Entscheidungen werden dann tendenziell zeitlich verzögert, an suboptimalen Orten oder nur zum Teil bearbeitet. Dies ist insbesondere bei bereichsübergreifenden Entscheidungen der Fall, weil hierfür wenig Klarheit über die Art und Weise besteht, wie entschieden wird, sondern wie eingangs illustriert, «(... )sowieso alles sehr viel über die informellen Netzwerke läuft (...)».

Die wesentliche Leistung des «bilateral-situativen Entscheidens» liegt trotz der hier skizzierten Herausforderungen in der Stabilisierung der Gesamtorganisation unter Anerkennung der relativ hohen Autonomie von einzelnen Teilbereichen und der damit verbundenen Verteiltheit von Entscheidungen. Gleichzeitig benötigt das «bilateral-situative Entscheiden» einige Voraussetzungen: Mit Blick auf die Gesamtorganisation braucht das bilateral-situative Entscheiden ausreichend finanzielle, personelle und zeitliche Ressourcen. Denn gerade organisationsweite Entscheidungen sind mit langwierigen $\mathrm{Ab}$ stimmungsprozessen verbunden.

Bilateral-situatives Entscheiden erfordert von den Beteiligten, dass sie in die informellen Netzwerke eines Spitals gut eingebunden sind, die jeweiligen Akteure, ihre Interessen und Beziehungen gut kennen. Insbesondere diejenigen, die keine Vertreter einer ärztlichen oder pflegerischen Profession sind, müssen sich darüber hinaus eine wohlwollende Re-

Abbildung 2

Bilateral-situatives Entscheiden.

Sowohl-als auch «bilateral-situatives Entscheiden» Ebene der Kliniken/Fachbereiche/Abteilungen vs. Ebene Gesamtspital

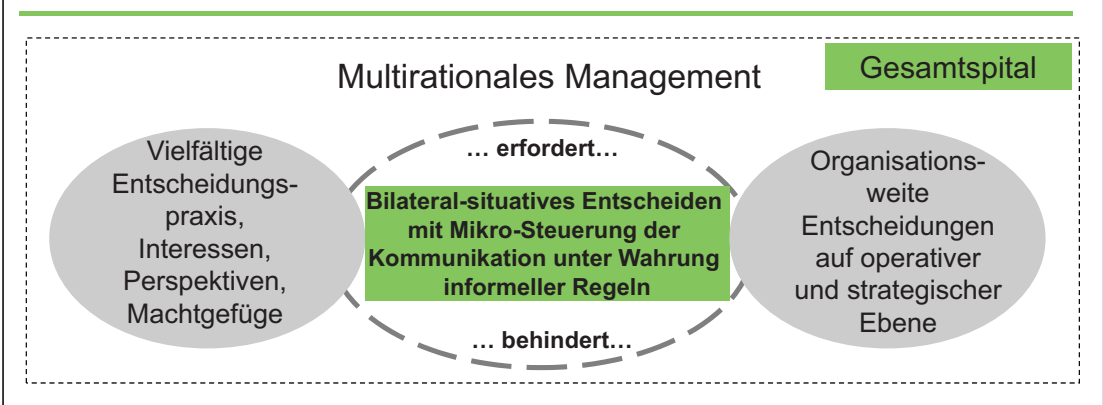

putation erarbeiten, so dass autonome Fachbereiche ihnen genügend Handlungsspielraum zugestehen. So äussert sich ein Klinikleiter über den Spitalleiter: «Bei ihm weiss man, dass er keinem was Böses will.» Dieser Vertrauensbeweis eröffnet Handlungsspielräume und legitimiert den Spitalleiter, der in diesem Fall keinen ärztlich-pflegerischen Hintergrund hat.

Zusammenfassend ist das bilateral-situative Entscheiden eine Variante, die sowohl Vielfältigkeit als auch organisationsweite Entscheidungen ermöglicht. Bilateral-situatives Entscheiden erlaubt es, die Paradoxie zu handhaben, und stabilisiert den Arbeitsalltag eines Spitals. Die Abbildung 2 enthält diesen Zusammenhang schematisch.

Jedoch ist das bilateral-situative Entscheiden an Voraussetzungen geknüpft, die derzeit schwinden. Zum einen steigen die organisationsweiten Entscheidungsnotwendigkeiten aufgrund von Spezialisierung innerhalb der Wertschöpfung an. Die Bearbeitung dieser Entscheidungsnotwendigkeiten wird durch die wahrgenommene Ressourcenverknappung verschärft. Zum anderen stellen sich für Spitäler zunehmend Fragen nach ihrer Positionierung gegenüber anderen Anbietern, nach ihrem Leistungsangebot und ihrer Vernetzung in Kooperationen mit anderen. Dabei wird es zunehmend auch darum gehen, sich von bestimmten Aktivitätsfeldern ganz zu trennen oder diese nicht mehr weiterzuentwickeln. Bei derartigen Fragestellungen zur internen Abstimmung für patientenzentrierte Abläufe und zur Positionierung des Spitals bei zunehmend wahrgenommener Ressourcenknappheit greift das bilateral-situative Entscheiden mitunter zu kurz.

Der folgende dritte Beitrag stellt angesichts der heutigen Entwicklung eine zweite Möglichkeit vor, wie mit der Paradoxie von Management im Spital umgegangen werden kann.

\section{Literatur}

1 Tuckermann H, Rüegg-Stürm J, Mitterlechner M. Zur Unmöglichkeit des Managements von Spitälern - die Paradoxie von Management. Schweiz Ärztezeitung. 2014;95(16/17):662-5.

2 Clegg S, Vieira da Cunha J, Pina e Cunha, M. Management paradoxes: A relational view. Human Relations. 2002;55(5):483-505.

3 Ganeri J. Indian Logic. In: Gabbay DM, Woods J (Eds.) Handbook of the History of Logic. Volume 1: Greek, Indian and Arabic Logic, 1 ed., Vol. 1:309-96. New York: Elsevier; 2004.

4 Smith W K,Lewis M W. Towards a theory of paradox: a dynamic equilibrium model of organizing. Academy of Management Review. 2011;36(2):381-401.

5 Denis JL, Lamothe L, Langley A. The Dynamics of Collective Leadership and Strategic Change in Pluralistic Organizations. Academy of Management Journal. 2001; 44(4): 800-37. 